\title{
CMEARTICLE
}

\section{Approach to the snoring child}

Yi Hua $\underline{\operatorname{Tan}}^{1,2}$, MMed, MRCPCH, Choon How $\underline{H o w}^{3,4}$, MMed, FCFP, Yoke Hwee $\underline{C h a n}^{2,5}$, MMed, MRCPCH, Oon Hoe $\underline{T e O h}^{1,2}$, MMed, MRCPCH

Five-year-old Thomas came to your clinic with his father for an upper respiratory tract infection. On examining him, you noticed a crowded pharynx with large and prominent tonsils. You had documented enlarged tonsils during his previous visits for other mild viral illnesses. This prompted you to ask further, and you discovered that the boy's elder sister in the same bedroom often complained of his loud snoring and frequent turning in bed during sleep.

\section{WHAT IS SNORING?}

Snoring is the harsh sound produced due to an obstruction to the smooth flow of air through the passages of the nose and the pharynx when the collapsible soft tissue structures in this area (i.e. tongue, soft palate, uvula, tonsils, adenoids and pharyngeal wall) strike one another and vibrate during breathing in sleep. Habitual snoring, which is defined as snoring for three or more nights in a week, may be a manifestation of underlying sleepdisordered breathing (SDB).

Obstructive SDB in childhood is defined as a syndrome of upper airway dysfunction during sleep, characterised by snoring and/or increased respiratory effort secondary to increased upper airway resistance and pharyngeal collapsibility. ${ }^{(1)}$ The spectrum of paediatric obstructive SDB, in increasing order of severity, comprises primary snoring (PS), upper airway resistance syndrome, obstructive hypoventilation and obstructive sleep apnoea (OSA).

PS involves snoring during sleep without abnormal ventilation or sleep disturbance. Upper airway resistance syndrome involves snoring during sleep with laboured breathing and frequent arousals with no observable obstructive events or changes in gas exchange. Obstructive hypoventilation is defined as continuous partial collapse of the pharyngeal airway during sleep with hypercapnia and hypoxaemia in the absence of cyclic discrete obstructive events. OSA involves recurrent episodes of prolonged partial or intermittent complete upper airway obstruction with resultant abnormal ventilation, gas exchanges abnormalities or sleep disruption.

This article discusses our suggested approach to children with snoring in the primary care setting, the risk factors and diagnosis of OSA, and the indications for referral to a paediatric sleep specialist.

\section{HOW RELEVANT IS THIS TO MY PRACTICE?}

Intermittent snoring is commonly observed in children during an intercurrent illness, with up to $28 \%$ of healthy children snoring occasionally, while habitual snoring affects $3 \%-12 \%$ of children. ${ }^{(2)}$
Although awareness about sleep and snoring is increasing in the general population, snoring is still mistakenly perceived by many parents to be benign and a reflection of good sleep, and therefore is seldom the presenting complaint for a consultation with a doctor. As the first-line healthcare provider for common acute paediatric conditions in children, primary care physicians are in the best position to screen and intervene early in cases of habitual snoring in children. This is important as the abnormal ventilation leading to hypoxaemia, increased work of breathing and sleep fragmentation from undiagnosed OSA can lead to significant health consequences.

Untreated OSA may lead to daytime somnolence; neurobehavioural, cognitive and functional impairment; and adversely affect learning, school performance and quality of life. A previous study demonstrated OSA in $18 \%$ of six-yearold children performing in the lowest $10 \%$ of their cohort and a subsequent improvement in their performance after adenotonsillectomy. ${ }^{(3)}$ OSA is also associated with significant cardiovascular and metabolic comorbidities. ${ }^{(4)}$ SDB has been shown to be associated with higher systolic blood pressures in school-going children. ${ }^{(5)}$ In the older paediatric cohort, adolescents with OSA were found to have a six-fold increase in the odds of developing metabolic syndrome compared to those without. ${ }^{(6)}$ Failure to thrive may be present in some children with OSA due to increased work of breathing during sleep and disruption of insulin-like growth factor binding protein-3 circulating level. Pulmonary hypertension and cor pulmonale are potential complications of severe untreated OSA.

The prevalence of OSA is $1.2 \%-5.7 \%$ in children of all ages. ${ }^{(7)}$ Both boys and girls are equally affected before puberty, with the peak incidence of OSA occurring at the age of 2-8 years, during which tonsillar and/or adenoidal hypertrophy is common. With increasing societal affluence and the resultant rising prevalence of obesity, there is now a second peak incidence in children aged above eight years, contributing to the rise in paediatric OSA. ${ }^{(8)}$ After puberty, there is a male preponderance for OSA.$^{(9)}$

\footnotetext{
${ }^{1}$ Respiratory Medicine Service, Department of Pediatrics, KK Women's and Children's Hospital, ${ }^{2}$ Paediatrics Academic Clinical Programme, SingHealth Duke-NUS Academic Medical Centre, ${ }^{3} \mathrm{C}$ are and Health Integration Division, Changi General Hospital, ${ }^{4}$ Family Medicine Academic Clinical Programme, SingHealth Duke-NUS Academic Medical Centre, 5Division of Medicine, KK Women's and Children's Hospital, Singapore

Correspondence: Dr Tan Yi Hua, Associate Consultant, Respiratory Medicine Service, Department of Paediatrics, KK Women's and Children's Hospital, 100 Bukit Timah Road, Singapore 229899. tan.yi.hua@singhealth.com.sg
} 
Across the entire paediatric age range, OSA is also more common in children with allergic rhinitis or chronic nasal congestion/ obstruction. There are many other risk factors for OSA besides these three common ones. They involve conditions that affect the size, collapsibility and neural control of the upper airway (e.g. trisomy 21, Pierre Robin sequence, laryngomalacia and achondroplasia) but are not within the scope of this article. Box 1 describes the common risk factors of OSA.

Early diagnosis and intervention is paramount to prevent unwanted physical, cognitive and developmental consequences in children with untreated OSA. With increasing awareness of the importance of sleep and snoring through public health education in the mass media, primary care providers will be seeing more parents who are concerned about their child's sleep and snoring, and should be ready to provide evidence-based, up-to-date advice and management.

\section{WHAT CAN I DO IN MY PRACTICE?}

Besides nutrition and physical activity, sleep is an important component that contributes to the healthy growth and development of a child. It is therefore recommended that primary care providers screen opportunistically for sleep problems in children. In a busy primary care setting, the screening can be done using the simple 'BEARS' sleep screening tool shown in Table I. ${ }^{(10)}$ If the child is found to have habitual snoring or excessive daytime sleepiness, a more detailed history and targeted physical examination should be performed to evaluate for OSA.

It is important to be aware of the risk factors for OSA in children. If the child falls into the age range with higher prevalence of OSA or is noted to have any of the common risk factors for OSA in children (tonsillar and/or adenoidal hypertrophy, obesity and allergic rhinitis/chronic nasal obstruction) on physical examination, further history and physical examination should also be performed to evaluate for OSA.

\section{History taking}

Symptoms of OSA may be difficult to elicit but can be largely divided into daytime and nocturnal symptoms. Nocturnal symptoms include habitual snoring, mouth breathing, witnessed apnoea, snorting or gasping during sleep, restless sleep, increased effort in breathing, excessive sweating, primary or secondary nocturnal enuresis, unusual sleeping positions (e.g. hyperextended neck) and, in severe cases, observed cyanosis. Typical daytime symptoms include unrefreshed sleep, morning headaches and daytime sleepiness. However, not all children present with typical daytime symptoms; some may exhibit hyperactivity, attention deficit, behavioural problems and academic deterioration.

Many parents do not sleep with their child or may not have paid attention to the details of their child's sleep before concerns with snoring or OSA risk factors are identified at the initial consultation. They can be informed of the symptoms and signs to look out for, and later check with family members who share a bedroom with the child, or observe the child during sleep themselves. In some cases, teachers and the child's peers may be the ones who notice the snoring during school camps and

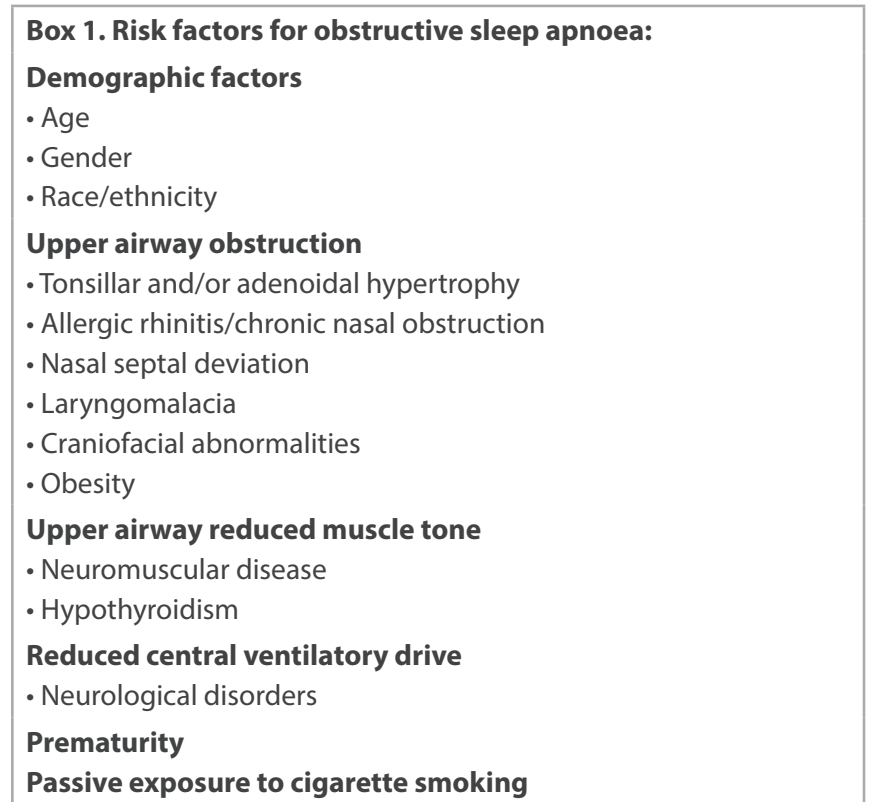

provide this history. OSA is usually worse during REM (rapid eye movement) sleep, which predominates in the later half of the night. Hence, parents should be advised to check for snoring and other symptoms of OSA in the later half of the night if they do not observe snoring at sleep onset. The perception of loudness and severity of snoring, work of breathing, and even gasping/ choking can be subjective. Parents can be advised to take a video recording of their child's sleep for review by the primary care provider at the next clinic consult, if there is suspicion that the parent may be under-reporting or not recognising the symptoms and signs.

It is important to assess and document the impact of the OSA on the child and family members, as this can help them to appreciate how their lives or health has been affected, and to be more receptive to the recommended treatment or referral. This also serves as a baseline for comparison for follow-up, especially after treatment. For example, a child initially noted to be always sleepy in class may have his teacher reporting significant improvement after treatment. Sometimes, family members sharing the bedroom may report better sleep for themselves as the child's snoring improves.

\section{Physical examination}

A targeted physical examination helps to ascertain the likelihood and/or severity of the OSA. Physical examination can be divided into:

- General examination, including growth parameters and facial features, with (a) weight, height and body mass index for assessment of obesity; (b) syndromic features (e.g. trisomy 21), mid-face hypoplasia or retrognathia/ micrognathia, which may limit upper airway size; and (c) pectus excavatum, growth failure and loud second heart sound (sign of pulmonary hypertension), which suggest long-standing, significant OSA

- Nasal examination for (a) features of allergic rhinitis, e.g. turbinate hypertrophy; and (b) nasal septum deviation, 
Table I. The 'BEARS' sleep screening tool.

\begin{tabular}{|c|c|c|c|}
\hline & Pre-school (2-5 yr) & School-aged (6-12 yr) & Adolescent (13-18 yr) \\
\hline $\begin{array}{l}\text { Bedtime } \\
\text { problems }\end{array}$ & $\begin{array}{l}\text { P: Does your child have any } \\
\text { problems going to bed? Falling } \\
\text { asleep? }\end{array}$ & $\begin{array}{l}\text { P: Does your child have any problems at bedtime? } \\
\text { C: Do you have any problems going to bed? }\end{array}$ & $\begin{array}{l}\text { C: Do you have any problems } \\
\text { falling asleep at bedtime? }\end{array}$ \\
\hline $\begin{array}{l}\text { Excessive } \\
\text { daytime } \\
\text { sleepiness }\end{array}$ & $\begin{array}{l}\text { P: Does your child seem overly tired } \\
\text { or sleepy a lot during the day? } \\
\text { Does your child still take naps? }\end{array}$ & $\begin{array}{l}\text { P: Does your child have difficulty waking in the } \\
\text { morning, seem sleepy during the day or take naps? } \\
\text { C: Do you feel tired a lot? }\end{array}$ & $\begin{array}{l}\text { C: Do you feel sleepy a lot } \\
\text { during the day? In school? } \\
\text { While driving? }\end{array}$ \\
\hline $\begin{array}{l}\text { Awakenings at } \\
\text { night }\end{array}$ & $\begin{array}{l}\text { P: Does your child wake up a lot } \\
\text { at night? }\end{array}$ & $\begin{array}{l}\text { P: Does your child seem to wake up a lot at night? } \\
\text { Any sleepwalking or nightmares? } \\
\text { C: Do you wake up a lot at night? Have trouble } \\
\text { getting back to sleep? }\end{array}$ & $\begin{array}{l}\text { C: Do you wake up a lot at } \\
\text { night? Have trouble getting } \\
\text { back to sleep? }\end{array}$ \\
\hline $\begin{array}{l}\text { Regularity and } \\
\text { duration of sleep }\end{array}$ & $\begin{array}{l}\text { P: Does your child have a regular } \\
\text { bedtime and wake time? What } \\
\text { are they? }\end{array}$ & $\begin{array}{l}\text { P: What time does your child go to bed and get up } \\
\text { on schooldays? Weekends? Do you think he/she } \\
\text { is getting enough sleep? }\end{array}$ & $\begin{array}{l}\text { C: What time do you usually } \\
\text { go to bed on school nights? } \\
\text { Weekends? } \\
\text { How much sleep do you } \\
\text { usually get? }\end{array}$ \\
\hline $\begin{array}{l}\text { Sleep-disordered } \\
\text { breathing }\end{array}$ & $\begin{array}{l}\text { P: Does your child snore a lot or } \\
\text { have difficulty breathing at night? }\end{array}$ & $\begin{array}{l}\text { P: Does your child have loud snoring or nightly } \\
\text { snoring or any breathing difficulties at night? }\end{array}$ & $\begin{array}{l}\text { P: Does your teenager snore } \\
\text { loudly or nightly? }\end{array}$ \\
\hline
\end{tabular}

C: child; P: parent

presence of mouth breathing and/or hyponasal speech suggesting nasal obstruction and/or adenoidal hypertrophy

- Oral examination for (a) tonsillar hypertrophy, which can be graded using the Brodsky grading scale to allow an objective initial and follow-up assessment; and (b) large tongue, higharched palate, malocclusion, small oropharyngeal space and high Mallampati score, which may indicate limitation of upper airway size.

\section{Investigations}

The gold standard investigation for paediatric OSA is overnight polysomnography (PSG) performed in a sleep laboratory in attendance by a sleep technologist. Unlike in the adult setting, other alternative investigations such as home unattended PSG, other multi-channel home sleep apnoea tests, and overnight home pulse oximetry are not as well validated, and have limitations with sensitivity and specificity in the evaluation of children with suspected OSA. While a PSG is generally preferred, there are real-world issues, including high costs, limited availability, long wait time, and tolerability of the many sensors placed on the body during PSG in very young children, especially those with special needs. In addition, automated PSG reporting is not recommended for children, as it has been shown to be inaccurate. Hence, it is important that the child sees a paediatric sleep specialist for evaluation and undergoes the PSG in a paediatric sleep laboratory, after which the findings are interpreted using paediatric-specific criteria. For children in whom a PSG is not feasible, the paediatric sleep specialist will discuss with the parent about alternative investigations best suited to the child's situation and interpret the results, taking into account the known limitations of the investigation.

\section{Management}

Primary care providers can consider a trial of intranasal corticosteroids and/or leukotriene antagonists in paediatric patients with habitual snoring only or habitual snoring with few symptoms/signs of OSA. This group of children are most likely to either have PS or mild OSA. Despite the absence of hypoxaemia and sleep arousals, PS may still be associated with cognitive and behavioural impairment in some children, ${ }^{(11)}$ so medical treatment should not be neglected. Children with mild OSA should be treated with intranasal corticosteroids and/or leukotriene antagonist as first-line management. A meta-analysis of treatment of paediatric OSA with intranasal corticosteroids alone showed a mild improvement of the Apnoea-Hypopnoea Index (AHI), a PSG index used to grade the severity of OSA, of 1.1. (12) Another study looking at the effect of combined intranasal corticosteroids with leukotriene antagonists showed normalisation of the $\mathrm{AHI}$ in up to $62 \%$ of subjects. ${ }^{(13)}$ If there is minimal or partial resolution of symptoms after a trial of medical treatment, a referral should be made to a paediatric sleep specialist for further evaluation. If there are multiple symptoms in the patient's history or worrisome examination findings suggestive of significant long-standing OSA, a referral should be made to a paediatric sleep specialist immediately. Treatment of paediatric OSA in a tertiary hospital is coordinated by a multidisciplinary team, including a paediatric sleep specialist (usually pulmonologist), paediatric otolaryngologist and paediatric orthodontist. Engaging the parents about their perception of snoring, OSA and treatment (e.g. adenotonsillectomy) by medical providers at every visit would help to identify misconceptions that can be addressed accordingly, and facilitate timely treatment and management for the child.

Treatment of paediatric OSA is largely dependent on the underlying cause and each individual's risk factors. In paediatric patients with enlarged tonsils and/or adenoids, surgery would usually be the first-line treatment after medical treatment. The prevalence of residual paediatric OSA after adenotonsillectomy ranged from $34 \%$ to $87 \%$ in a systematic review by Lee at 
al, depending on the characteristics of the study population and $\mathrm{AHI}$ definition for residual OSA. Obese children are more likely to have residual OSA than children with normal body mass index. ${ }^{(14)}$ Other risk factors for residual OSA in children following adenotonsillectomy include predisposing syndromes, neuromuscular/neurological disorders, craniofacial or orthodontic abnormalities. Many parents have concerns over general anaesthesia and impairment of immunity with adenotonsillectomy. They should be reassured that for children with moderate to severe OSA due to adenotonsillar hypertrophy not responding to medical therapy, the benefit of adenotonsillectomy likely outweighs the risk of the general anaesthesia and surgery, and that the surgery does not impair immunity in children with a normal immune system. Obese patients will need to lose weight through healthy eating and regular exercise. They can also be referred to weight management programmes for obese children. In children who are not suitable candidates for surgery, or who have significant residual OSA post surgery, continuous positive airway pressure (CPAP) therapy during sleep may be recommended. Children on CPAP treatment would need regular long-term follow-up with a paediatric sleep specialist, as there are often adherence issues and concerns about restriction of mid-face development with long-term use. A small group of children may benefit from an orthodontic assessment and management for their OSA.

In OSA that is multifactorial in cause, management would require a multidisciplinary approach. The importance of good sleep hygiene, lifestyle modifications and adherence to medical therapy for allergic rhinitis need to be regularly emphasised. Documenting patients and their parents' reports of significant improvements in symptoms and signs of OSA at every visit is useful to monitor patients' progress. While adenotonsillectomy is effective in resolving OSA for most children, OSA may recur later for a group of children from adenoidal regrowth, neglect of allergic rhinitis management or development/worsening of obesity. Primary care providers are in a good position to reinforce healthy sleep, lifestyle and management adherence advice, and also to follow up with the child for recurrence of OSA during primary care visits.

\section{TAKE HOME MESSAGES}

1. Intermittent snoring is common in children but habitual snoring, which affects about $3 \%-12 \%$ of children, is of concern and needs further evaluation.

2. It is important to identify OSA in children, as it may lead to physical, developmental and cognitive consequences for the child if left untreated.

3. The 'BEARS' sleep screening tool is useful in the primary care setting to identify sleep problems, including OSA, in children.

4. Primary care providers should be alert to the three most common risk factors for OSA in children (obesity, tonsillar and/or adenoidal hypertrophy, and allergic rhinitis/chronic nasal obstruction) and screen for snoring and symptoms/ signs of OSA if these are present.
5. Children with habitual snoring only, or habitual snoring with few symptoms/signs of OSA, may benefit from a trial of intranasal corticosteroids and/or leukotriene antagonists before referral to a paediatric sleep specialist.

6. Overnight inpatient PSG is the gold standard for definitive diagnosis of OSA in children. This should be performed in a paediatric sleep laboratory and interpreted with reference to paediatric-specific criteria.

7. The most common surgical treatment for OSA in children is tonsillectomy and/or adenoidectomy.

8. OSA may recur (or persist) despite treatment, especially in children with underlying risk factors mentioned above. Primary care doctors can play an important co-management role in the follow-up and screening for recurrent/residual OSA after initial diagnosis and management by a paediatric sleep specialist.

On examination, you found that Thomas was well-thrived and not obese but had swollen nasal turbinates. You started him on intranasal corticosteroids for his allergic rhinitis. His father reported improved but persistent habitual snoring with mouth breathing. You referred Thomas to a paediatric sleep specialist. On polysomnography, moderate obstructive sleep apnoea (OSA) was diagnosed, and he was referred to a paediatric otolaryngologist for adenotonsillectomy. With both medical and surgical therapy, Thomas' OSA symptoms had resolved completely when you reviewed him in your clinic nine months later. Both his father and teachers noticed that he was more active and better able to concentrate in class.

ABSTRACT Sleep is an important component in a child's growth and development. Snoring is common in children and often perceived as benign, but habitual snoring may be an indication of obstructive sleep apnoea (OSA). OSA can have health, developmental and cognitive consequences. The three common risk factors for paediatric OSA are tonsillar and/or adenoidal hypertrophy, obesity and allergic rhinitis. Primary care providers are well-placed to identify children at risk by screening for habitual snoring and associated OSA risk factors during routine consultations. Physician awareness of OSA symptoms/signs facilitates diagnosis, management and referral decisions. A trial of medical treatment may be considered for habitual snoring with mild symptoms/signs before referral. Overnight polysomnography is the gold standard investigation utilised by paediatric sleep specialists to diagnose OSA. Adenotonsillectomy is the first-line management for OSA with adenotonsillar hypertrophy, but residual/recurrent OSA may occur, so follow-up by primary care providers is important after surgery.

Keywords: child, obstructive, sleep apnoea, snoring 


\section{REFERENCES}

1. Kaditis AG, Alonso Alvarez ML, Boudewyns A, et al. Obstructive sleep disordered breathing in 2- to 18-year-old children: diagnosis and management. Eur Respir J 2016; 47:69-94.

2. Chng SY, Goh DY, Wang XS, Tan TN, Ong NB. Snoring and atopic disease: strong association. Pediatr Pulmonol 2004; 38:210-6.

3. Gozal D. Sleep-disordered breathing and school performance in children. Pediatrics 1998; 102:616-20.

4. Ng DKK, Huang YS, Teoh OH, et al. The Asian Paediatric Pulmonology Society (APPS) position statement on childhood obstructive sleep apnea syndrome. Pediatr Respirol Crit Care Med 2017; 1:26-38.

5. Bixler EO, Vgontzas AN, Lin HM, et al. Blood pressure associated with sleepdisordered breathing in a population sample of children. Hypertension 2008 52:841-6

6. Redline S, Storfer-Isser A, Rosen CL, et al. Association between metabolic syndrome and sleep-disordered breathing in adolescents. Am J Respir Crit Care Med 2007: 176:401-8.

7. DelRosso LM. Epidemiology and diagnosis of pediatric obstructive sleep apnea.
Curr Probl Pediatr Adolesc Health Care 2016; 46:2-6.

8. Chay OM, Goh A, Abisheganaden J, et al. Obstructive sleep apnea syndrome in obese Singapore children. Pediatr Pulmonol 2000; 29:284-90.

9. Bandla $\mathrm{H}_{1} \mathrm{D}^{\prime}$ Andrea LA. Natural history and management of pediatric obstructive sleep apnea-emerging concepts. Sleep 2015; 38:11-2.

10. Owens JA, Dalzell V. Use of the 'BEARS' sleep screening tool in a pediatric residents' continuity clinic: a pilot study. Sleep Med 2005; 6:63-9.

11. Biggs SN, Nixon GM, Horne RS. The conundrum of primary snoring in children: what are we missing in regards to cognitive and behavioural morbidity? Sleep Med Rev 2014; 18:463-75.

12. Liu HT, Lin YC, Kuan YC, et al. Intranasal corticosteroid therapy in the treatment of obstructive sleep apnea: a meta-analysis of randomized controlled trials. Am J Rhinol Allergy 2016; 30:215-21.

13. Kheirandish-Gozal L, Bhattacharjee R, Bandla HPR, Gozal D. Antiinflammatory therapy outcomes for mild OSA in children. Chest 2014; 146:88-95.

14. Lee $\mathrm{CH}$, Hsu WC, Chang WH, Lin MT, Kang KT. Polysomnographic findings after adenotonsillectomy for obstructive sleep apnoea in obese and non-obese children: a systematic review and meta-analysis. Clin Otolaryngol 2016; 41:498-510. 


\section{SINGAPORE MEDICAL COUNCIL CATEGORY 3B CME PROGRAMME} (Code SMJ 202004A)

1. Snoring is any sound produced from the nose and the pharynx during breathing in sleep.

2. Up to $28 \%$ of healthy children snore occasionally, while habitual snoring affects $3 \%-12 \%$ of children.

3. Habitual snoring, which is defined as snoring for two or more nights in a week, may be a manifestation of underlying sleep-disordered breathing.

4. Upper airway resistance syndrome, obstructive hypoventilation and obstructive sleep apnoea (OSA) are difficult to differentiate and diagnose from a structured history or clinical examination.

5. Snoring observed in children during an acute illness is a strong suggestion of obstructive sleep-disordered breathing.

6. Awareness about sleep and snoring is increasing in the general population, and most parents today know that snoring is benign and a reflection of good sleep.

7. Primary care providers are in the best position to screen and intervene early to reduce increased work of breathing, sleep fragmentation and consequences of undiagnosed OSA.

8. A previous study demonstrated OSA in $18 \%$ of six-year-old children performing in the lowest $10 \%$ of their cohort and a subsequent improvement in their performance after adenotonsillectomy.

9. OSA in children, unlike in adults, is not associated with significant cardiovascular and metabolic comorbidities.

10. The prevalence of OSA is $1.2 \%-5.7 \%$ in children, with the peak incidence occurring between the ages of two and eight years.

11. With increasing societal affluence and the resultant rising prevalence of obesity, there is now a second peak in OSA incidence in children, especially in girls aged above eight years.

12. OSA is more common in children with allergic rhinitis or chronic nasal congestion.

13. The 'BEARS' sleep screening tool is a structured tool to detect habitual snoring or excessive daytime sleepiness, such that a more detailed history and targeted physical examination can be performed to evaluate for OSA.

14. The symptoms of paediatric OSA may be difficult to elicit, as they are all nocturnal symptoms such as witnessed apnoea, snorting or gasping during sleep, restless sleep, primary nocturnal enuresis and, in severe cases, observed cyanosis.

15. Many parents may not have paid attention to their child's sleep and can be informed of the symptoms and signs to look out for, or can consider taking a video recording of their child's sleep for review at the next clinic consult.

16. As OSA is usually worse during REM (rapid eye movement) sleep, which predominates in the later half of the night, parents should be advised to check for symptoms at both the onset of sleep and in the later half of the night.

17. Primary care providers can consider a trial of intranasal corticosteroids and/or leukotriene antagonists in paediatric patients with habitual snoring only or habitual snoring with few symptoms/signs of OSA.

18. Treatment of OSA needs to be individualised, with surgical removal of enlarged tonsils and/or adenoids being a common first-line treatment after failure of a trial of medical treatment.

19. The greatest risk for children with a normal immune system and moderate to severe OSA after adenotonsillectomy is impaired immunity.

20. While adenotonsillectomy is effective in resolving OSA for most children, OSA may recur later from adenoidal regrowth, neglect of allergic rhinitis management or development/worsening of obesity.

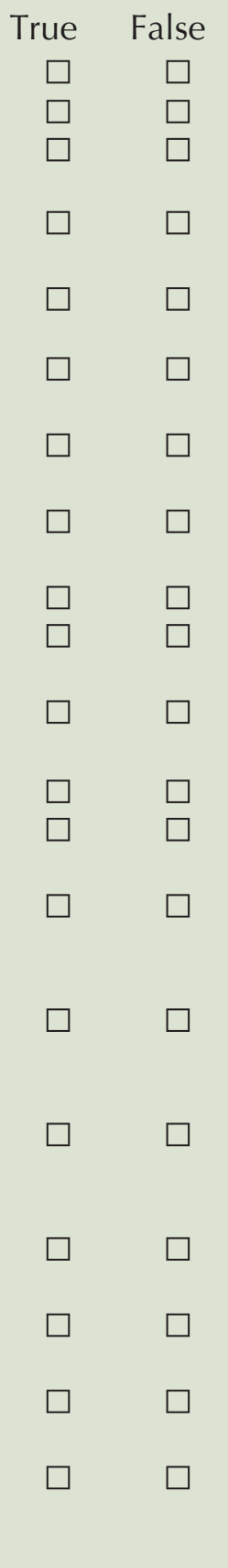

\section{Doctor's particulars:}

Name in full:

Specialty:

MCR no.:

Email:

\footnotetext{
SUBMISSION INSTRUCTIONS:

Visit the SMJ website: http://www.smj.org.sg/current-issue and select the appropriate quiz. You will be redirected to the SMA login page.

For SMA member: 1) Log in with your username and password (if you do not know your password, please click on 'Forgot your password?'). (2) Select your answers for each quiz and click 'Submit'.

For non-SMA member: (1) Create an SMJ CME account, or log in with your SMJ CME username and password (for returning users). (2) Make payment of SGD 21.40 (inclusive of $7 \%$ GST) via PayPal to access this month's quizzes. (3) Select your answers for each quiz and click 'Submit'.

RESULTS:

(1) Answers will be published online in the SMJ June 2020 issue. (2) The MCR numbers of successful candidates will be posted online at the SMJ website by 9 June 2020. (3) Passing mark is $60 \%$. No mark will be deducted for incorrect answers. (4) The SMJ editorial office will submit the list of successful candidates to the Singapore Medical Council. (5) One CME point is awarded for successful candidates. (6) SMC credits CME points according to the month of publication of the CME article (i.e. points awarded for a quiz published in the April 2020 issue will be credited for the month of April 2020, even if the deadline is in June 2020).

Deadline for submission (April 2020 SMJ 3B CME programme): 12 noon, 2 June 2020.
} 